University of Wollongong

Research Online

Faculty of Informatics - Papers (Archive)

Faculty of Engineering and Information

Sciences

July 2004

\title{
The effect of imperfect carrier recovery on the performance of the diversity antenna selection technique in wireless channels utilizing DSTM
}

\author{
Le Chung Tran \\ University of Wollongong, Ictran@uow.edu.au \\ Tadeusz A. Wysocki \\ University of Wollongong, wysocki@uow.edu.au \\ Alfred Mertins \\ University of Oldenburg, Germany, mertins@uow.edu.au
}

Follow this and additional works at: https://ro.uow.edu.au/infopapers

Part of the Physical Sciences and Mathematics Commons

\section{Recommended Citation}

Tran, Le Chung; Wysocki, Tadeusz A.; and Mertins, Alfred: The effect of imperfect carrier recovery on the performance of the diversity antenna selection technique in wireless channels utilizing DSTM 2004.

https://ro.uow.edu.au/infopapers/108

Research Online is the open access institutional repository for the University of Wollongong. For further information contact the UOW Library: research-pubs@uow.edu.au 


\title{
The effect of imperfect carrier recovery on the performance of the diversity antenna selection technique in wireless channels utilizing DSTM
}

\author{
Abstract \\ This paper examines the effect of imperfect carrier phase/frequency recovery at the receiver on the bit \\ error performance of our diversity antenna selection technique (AST) proposed for channels utilizing \\ differential space-time modulation (DSTM). The tolerance of differential detection associated with the \\ proposed AST to phase/frequency errors is then analyzed.

\section{Keywords} \\ frequency modulation, receiving antennas, space-time codes

\section{Disciplines} \\ Physical Sciences and Mathematics

\section{Publication Details} \\ This paper originally appeared as: Tran, LC, Wysocki, TA \& Mertins, A, The effect of imperfect carrier \\ recovery on the performance of the diversity antenna selection technique in wireless channels utilizing \\ DSTM, 2nd International Conference on Information Technology: Research and Education, 28 June-1 July \\ 2004, 15-18. Copyright IEEE 2004.
}




\section{The Effect of Imperfect Carrier Recovery on the Performance of the Diversity Antenna Selection Technique in Wireless Channels Utilizing DSTM}

\author{
Le Chung Tran, Tadeusz A. Wysocki, Jennifer Seberry \\ University of Wollongong \\ Northfields Avenue, NSW 2522, Australia \\ Email: lct71,wysocki,jennie@uow.edu.au
}

\author{
Alfred Mertins \\ University of Oldenburg \\ 26111 Oldenburg, Germany \\ Email: alfred.mertins@uni-oldenburg.de
}

\begin{abstract}
This paper examines the effect of imperfect carrier phase/frequency recovery at the receiver on the bit error performance of our diversity antenna selection technique (AST) proposed for channels utilizing Differential Space-Time Modulation (DSTM). The tolerance of differential detection associated with the proposed AST to phase/frequency errors is then analyzed. ...
\end{abstract}

\section{INTRODUCTION}

The combination between the diversity of space-time codes and a closed loop diversity antenna selection technique (AST) using a feedback loop in coherent detection has been intensively examined in literature, such as [1], [2], [3], [4], [5]. However, ASTs for channels utilizing Differential Space-Time Modulation (DSTM) are not so widely considered. In [6] and [7], we propose ASTs for such the channels, where we assume that carrier phase/frequency (or just phase, for short, except when it is clearly stated) recovery is perfect. Simulation results show that DSTM associated with the proposed ASTs provides much better bit error performance than that without ASTs, and, at high signal-to-noise ratios ( $S N R \mathrm{~s})$, even better than coherent detection without ASTs [6], [7]. In fact, phase recovery errors always exist, which degrade the performance of the proposed ASTs. Phase errors may occur due to the difference between the frequency of the local oscillators at the transmitter and the receiver, and/or due to the Doppler frequency-shift effect in fast fading channels. Therefore, examining the effect of imperfect phase recovery on the performance of the proposed ASTs and, if possible, proposing solutions to overcome this problem are specially important. It is noted that, the phrase "antenna selection" is sometimes used in this paper instead of the phrase "our proposed antenna selection technique" for short.

In Section II of this paper, the examination of the effect of phase recovery errors on the performance of our proposed AST called the general N-out-of-M AST [6], [7] is carried out. Simulation results and discussion on the sensitivity to phase errors, as well as, on the error tolerance of DSTM with and without our AST are presented in Section III. The paper is concluded by Section IV.
II. EFFECT OF PHASE ERRORS ON THE PERFORMANCE OF THE PROPOSED ANTENNA SELECTION TECHNIQUE

In this paper, the DSTM based on the Alamouti code [8], or the Alamouti DSTM, for short, is examined. It is trivial to generalize the consideration mentioned here for other spacetime block codes, and therefore, we do not deal with this task here. In addition, we concentrate only on the general N-outof-M AST. The other proposed ASTs mentioned in [7] are the different particular cases of this AST. We assume here that, due to imperfect recovery, the initial phase error for each data segment ${ }^{1}$ comprising $\mathcal{L}$ symbol time slots is $\Delta \phi_{0}$ (radians). Meanwhile, non-initial phase errors are assumed to cumulate by adding a constant volume $\Delta \phi$ (radians) per symbol time slot to the phase of received signals. It means that the signal received in the $k^{\text {th }}(k=1 \ldots \mathcal{L})$ symbol time slot in the considered data segment is now multiplied with $e^{i\left[(k-1) \Delta \phi+\Delta \phi_{0}\right]}$. However, our simulation results show that the bit error performance of DSTM does not depend on the initial phase error $\Delta \phi_{0}$. This is interpreted as follows. Like the differential phase shift keying (DPSK) demodulation, where the demodulation of signals only depends on the relative phase difference between consecutive signals, not on the absolute phase of each signal, the bit error performance of DSTM does not depend on the initial phase error $\Delta \phi_{0}$, but depends on non-initial phase errors. Therefore, in the rest of this paper, we omit the initial phase error and only examine the effect of non-initial phase errors. Additionally, if we denote $\Delta f$ to be the frequency error per symbol time slot, it follows that:

$$
\Delta \phi=2 \pi \Delta f T_{s}=2 \pi \overline{\Delta f}
$$

where $T_{s}$ is the period of symbol time slots and $\overline{\Delta f}=\Delta f T_{s}$ is called the equivalent frequency error, which is the frequency error $\Delta f$ normalized by the frequency of transmitted symbols $F_{s}=\frac{1}{T_{s}}$.

First, we examine the case of the Alamouti DSTM without antenna selection. The most common scenario is that the

\footnotetext{
${ }^{1}$ By using the term "data segment" here (the same as "frame" in [6] and [7]) , the authors do not mean channels are quasi-flat fading. The readers may refer to [7] for more details.
} 
considered system comprises two transmit and one receive antennas. Again, it is trivial to generalize the consideration here for systems with an arbitrary number of transmit and/or receive antennas. As mentioned in [6], [7], in all DSTM techniques proposed in literature so far, it is required that the transmission gains are constant during at least two adjacent code blocks, which, for simplicity, we call a data segment. ${ }^{2}$ Therefore, we assumed here that the transmission gains are constant in a data segment of four symbol time slots corresponding to the time when the initial matrix $W_{0}=I_{2}$ ( $I$ is an identity matrix) and the next code block $W_{1}$ are transmitted. Clearly, this is the best case when phase recovery errors are taken into account, since, the larger the size of the considered data segment is, the larger the cumulative phase error in received signals is. Consequently, the bit error performance of differential detection degrades more. We denote $a_{j}$ and $n_{t j}(j=1,2 ; t=0,1)$ to be the transmission gain between the $j^{\text {th }}$ transmit antenna and the receive antenna, and the noise on this channel affecting the receiver during the $t^{\text {th }}$ transmission time, respectively (the $0^{\text {th }}$ transmission means the initial transmission). The expressions given below are straightforwardly derived according to the DSTM proposed in [10]. The readers may refer to [6], [7], or [10] for more details. The matrix $W_{1}$ transmitted after the initial transmission is:

$$
W_{1}=W_{0} Z_{1}=I_{2} Z_{1}=Z_{1}
$$

where $Z_{1}$ is defined as:

$$
Z_{1}=\frac{1}{\sqrt{2}}\left[\begin{array}{cc}
s_{1} & s_{2} \\
-s_{2}^{*} & s_{1}^{*}
\end{array}\right]
$$

The signals received during the initial transmission when $W_{0}=I_{2}$ is transmitted are given below (the second index of the received signals indicates the order of the corresponding symbol time slot):

$$
\begin{aligned}
& r_{01}=a_{1}+n_{01} \\
& r_{02}=a_{2} e^{i \Delta \phi}+n_{02}
\end{aligned}
$$

The signals received when the second code block $W_{1}=Z_{1}$ is transmitted are:

$$
\begin{aligned}
& r_{11}=\frac{1}{\sqrt{2}}\left(a_{1} s_{1}+a_{2} s_{2}\right) e^{i 2 \Delta \phi}+n_{11} \\
& r_{12}=\frac{1}{\sqrt{2}}\left(-a_{1} s_{2}^{*}+a_{2} s_{1}^{*}\right) e^{i 3 \Delta \phi}+n_{12}
\end{aligned}
$$

The above expressions are used in the simulation in the next section.

Next, we consider the Alamouti DSTM associated with our proposed AST. The general 2-out-of-4 AST is taken into account. As in all existing DSTM techniques, transmission

\footnotetext{
${ }^{2}$ This condition is not necessary if the linear prediction is utilized at the receiver, where multiple previous matrices of the received signals are used to predict the relation between the current transmission gain matrix and the previous ones [9]. As a result, the transmission gains are not necessary to be unchanged during at least two consecutive code blocks.
}

gains in the DSTM associated with our AST are also required to be constant during a data segment of at least two consecutive code blocks (i.e., six symbol time slots) corresponding to the time when the initial block $\tilde{W}_{0}=I_{4}$ and the next block $W_{1}$ are transmitted [6], [7]. Intuitively, it is clear that this case provides the best bit error performance when phase recovery errors are considered, as, the larger the size of the data segment is, the larger the cumulative phase error in received signals is. Similar to the case of differential detection without ASTs, we have the received signals during the initial transmission $\left(\tilde{W}_{0}=I_{4}\right.$ is transmitted) as given below:

$$
\begin{aligned}
& r_{01}=a_{1}+n_{01} \\
& r_{02}=a_{2} e^{i \Delta \phi}+n_{02} \\
& r_{03}=a_{3} e^{i 2 \Delta \phi}+n_{03} \\
& r_{04}=a_{4} e^{i 3 \Delta \phi}+n_{04}
\end{aligned}
$$

According to our general 2-out-of-4 AST proposed in [6], [7], we assume, without loss of generality, that, the transmit antennas which should be selected to transmit the considered data segment are the first and the second ones. In addition, as mentioned in [6], [7], the matrix $W_{1}$ transmitted after the initial matrix $\tilde{W}_{0}$ is calculated by multiplying the matrix $Z_{1}$ in (3) with a tacit default identity matrix $W_{0}=I_{2}$, and consequently, $W_{1}$ has the same expression as (2), i.e., $W_{1}=Z_{1}$. Hence, when the second code block $W_{1}$ is transmitted, the received signals are as follows:

$$
\begin{aligned}
& r_{11}=\frac{1}{\sqrt{2}}\left(a_{1} s_{1}+a_{2} s_{2}\right) e^{i 4 \Delta \phi}+n_{11} \\
& r_{12}=\frac{1}{\sqrt{2}}\left(-a_{1} s_{2}^{*}+a_{2} s_{1}^{*}\right) e^{i 5 \Delta \phi}+n_{12}
\end{aligned}
$$

\section{SIMULATION RESULTS}

In this section, the bit error rates (BER) of DSTM with and without our proposed AST versus the ratio between bit energy and noise energy $\left(E_{b} / N_{0}\right)$ are presented. The channel is assumed to be flat Rayleigh fading one. The Alamouti DSTM and the unitary QPSK signal constellation, where the power of the symbols in the constellation is one, are considered. The transmission gains and noise are assumed to be identically independently distributed (i.i.d.) complex Gaussian random variables with the distributions $\mathcal{C N}(0,1)$ and $\mathcal{C N}\left(0, \sigma^{2}\right)$, respectively.

The bit error performance of DSTM without antenna selection is shown in Figure 1(a). Again, we assume that the transmission gains remain unchanged in a data segment of four symbol time slots and change randomly from data segment to data segment. The performance of DSTM with our proposed AST is presented in Figure 1(b). In this simulation, the general 2-out-of-4 AST is used and we assume the transmission gains remain constant during a data segment comprising six symbol time slots. From (4), (5), (6) and (7) and the above assumptions, we realize that the received energy of each symbol during a symbol time slot is $1 / 2$, and, 


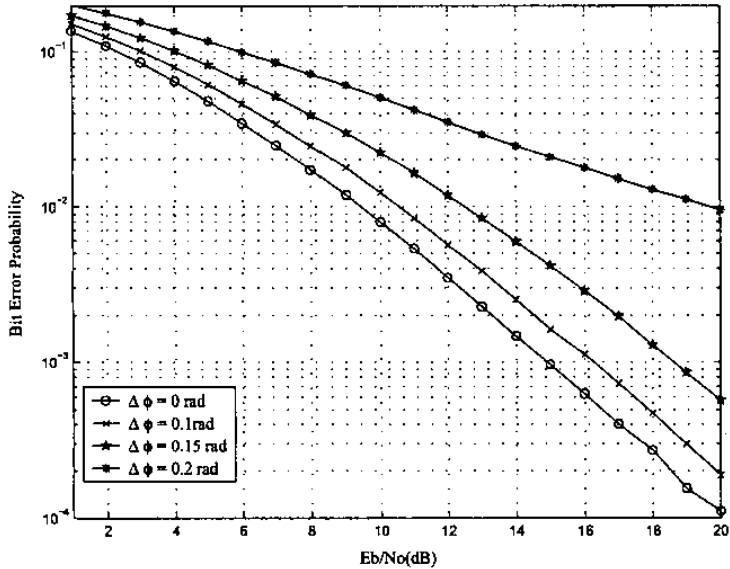

(a)

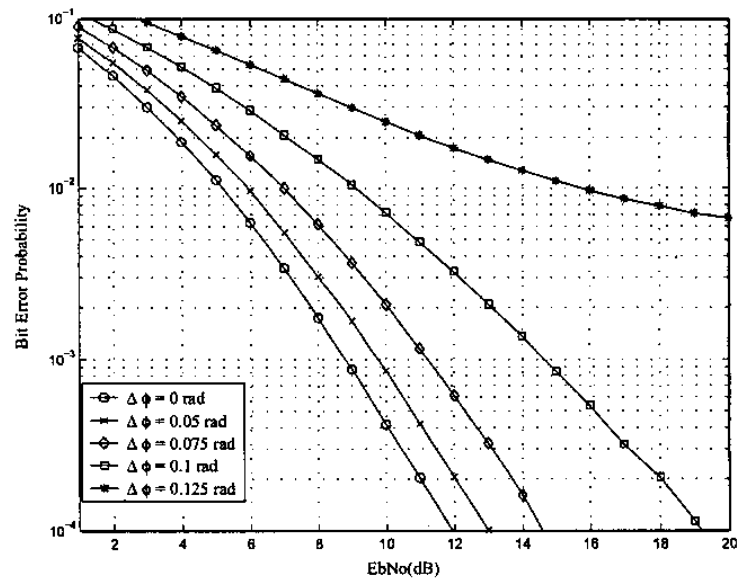

(b)

Fig. 1. The effect of imperfect phase recovery on the performance of DSTM (a): without, and (b): with our AST.

consequently, the energy per bit is $E_{b}=1 / 4$. Therefore, in both simulations, in order to compare the effect of different phase errors at a same given $E_{b} / N_{0}$, we assign the noise variance (or noise energy) to $\sigma^{2}=\frac{1}{4\left(E_{b} / N_{0}\right)}$.

We can see from Figure 1(a) that, when the AST is not utilized and the phase errors $\Delta \phi=0.1$ and $\Delta \phi=0.15$ rad exist, $E_{b} / N_{0}$ required to achieve the same $B E R=10^{-3}$ as in the case without phase errors $(\Delta \phi=0)$ is approximately 1.3 and $3.75 \mathrm{~dB}$ higher, respectively. The bit error performance of differential detection degrades rapidly for $\Delta \phi>0.15 \mathrm{rad}$. Likewise, in differential detection with our AST, $E_{b} / N_{0}$ is required 1 and $2.6 \mathrm{~dB}$ higher to achieve the same $B E R=10^{-4}$ as in the case without phase errors $(\Delta \phi=0)$, corresponding to the phase errors of 0.05 and $0.075 \mathrm{rad}$, respectively. The phase errors, which are greater than $0.075 \mathrm{rad}$, seriously degrade the performance of the proposed AST.

Therefore, DSTM associated with the proposed AST is more sensitive to phase errors than that without the AST. From the simulation results, we may deduce that the tolerance to phase errors in the cases with and without the proposed $\mathrm{AST}$ is $0.075 \mathrm{rad}$ (or $13.5^{\circ}$ ) and $0.15 \mathrm{rad}\left(\right.$ or $27^{\circ}$ ), respectively. Additionally, the figures show that DSTM accompanied with our proposed AST, in the cases of phase errors $\Delta \phi$ up to 0.1 rad corresponding to the four lower curves in Figure 1(b), has better bit error performance than DSTM without antenna selection no matter whether phase errors exist or not (see curves in Figure 1(a)). In other words, DSTM associated with our proposed AST mentioned in [6], [7] overwhelms that without antenna selection, even when some certain values of phase errors exist.

It is worth to note that Figures $1(a)$ and 1(b) themselves also present $B E R$ versus $E_{b} / N_{0}$ in the case of frequency recovery errors, since, frequency errors and phase errors follow the relation (1). It means the tolerance to equivalent frequency errors in DSTM with and without antenna selection is $\overline{\Delta f}=\frac{0.075}{2 \pi}=1.19 \times 10^{-2}$ and $\overline{\Delta f}=\frac{0.15}{2 \pi}=2.39 \times 10^{-2}$, respectively. In other words, the tolerance to frequency errors in the case of DSTM with and without our AST is approximately $1.19 \%$ and $2.39 \%$ of the symbol frequency $F_{s}=\frac{1}{T_{s}}$, respectively.

\section{CONCLUSION}

This paper examines the effect of the imperfect carrier phase/frequency recovery at the receiver on our AST proposed for channels using DSTM. Phase/frequency errors may be due to the difference between the frequency of the local oscillators in the transmitter and the receiver. They also may be caused by the Doppler frequency shift effect in the fast Rayleigh fading environment. As mentioned in [6], [7], DSTM combined with the proposed AST has much better performance than that without antenna selection, and, at high $S N R \mathrm{~s}$, even better than coherent detection without antenna selection. Simulation results in this paper provide further conclusions that, although DSTM associated with the proposed AST is more sensitive to imperfect carrier recovery, but for phase errors up to 0.1 rad, it still has better bit error performance than that without antenna selection (no matter whether phase errors exist or not). In addition, the tolerance to phase errors in the cases of DSTM with and without antenna selection is $0.075 \mathrm{rad}$ and $0.15 \mathrm{rad}$, respectively. Correspondingly, the tolerance to frequency errors in these cases is $1.19 \%$ and $2.39 \%$ of the symbol frequency $F_{s}$, respectively. The research on the effect of imperfect carrier recovery here opens other technical problems, which are the perspective examining issues, such as methods to compensate phase errors in order to enhance the 
performance of the proposed AST.

\section{ACKNOWLEDGEMENT}

L. C. Tran would like to thank Thanh Huyen Phan for very helpful comments on our papers.

\section{REFERENCES}

[1] A. Ghrayeb and T. M. Duman, "Performance analysis of MIMO systems with antenna selection over quasi-static fading channels", IEEE Trans. Veh. Technol., vol. 52, no. 2, pp. 281 - 288, Mar 2003.

[2] W. .H. Wong and E. G. Larson, "Orthogonal space-time block coding with antenna selection and power allocation", Electronics Lett., vol. 39 no. 4, pp. 379-381, Feb. 2003.

[3] D. Gore and A. Paulraj, "Space-time block coding with optimal antenna selection", Proc. IEEE Conference on Acoustics, Speech, and Signal Processing ICASSP'01, vol. 4, pp. 2441-2444, May 2001

[4] M. Katz, E. Tiirola and J. Ylitalo, "Combining space-time block coding with diversity antenna selection for improved downlink performance", Proc. IEEE Veh. Technol. Conf. VTC' 2001, vol. 1, 2001, pp. 178-182.

[5] L. C. Tran, T. A. Wysocki and A. Mertins, "Improved antenna selection technique to enhance the downlink performance of mobile communications systems", Proc. 7th International Symposium on Signal Processing and its Applications ISSPA'03, vol. 1, pp. 257-260, 1-4, July 2003.

[6] L. C. Tran and T. A. Wysocki, "Antenna selection scheme for wireless channels utilizing differential space-time modulation", Proc. 7th International Symposium on Digital Signal Processing and Communications Systems DSPCS 2003, pp. 452-457, Dec. 2003.

[7] L. C. Tran and T. A. Wysocki, "On some antenna selection techniques for wireless channels utilizing differential space-time modulation", IEEE Wireless Communications and Networking Conference WCNC 2004, Atlanta, Georgia, USA, 21-25, Mar. 2004.

[8] S. M. Alamouti, "A simple transmit diversity technique for wireless communications", IEEE J. Select. Areas Commun., vol. 16, no. 8, Oct. 1998.

[9] A. Song and X. G. Xia, "Decision feedback differential detection for differential orthogonal space-time modulation with ASPK signals over frequency-non-selective fading channels", IEEE International Conf. Commun. ICC'03, vol. 2, pp. 1253-1257, May 2003.

[10] G. Ganesan and P. Stoica, "Differential modulation using space-time block codes", IEEE Sign. Process. Lett., vol. 9, no. 2, pp. 57-60, Feb. 2002. 